\title{
Sustainable investment: A new landscape
}

Written by: Monika Dutt, Senior Investment Specialist at Vanguard

Last update: 17 August 2020

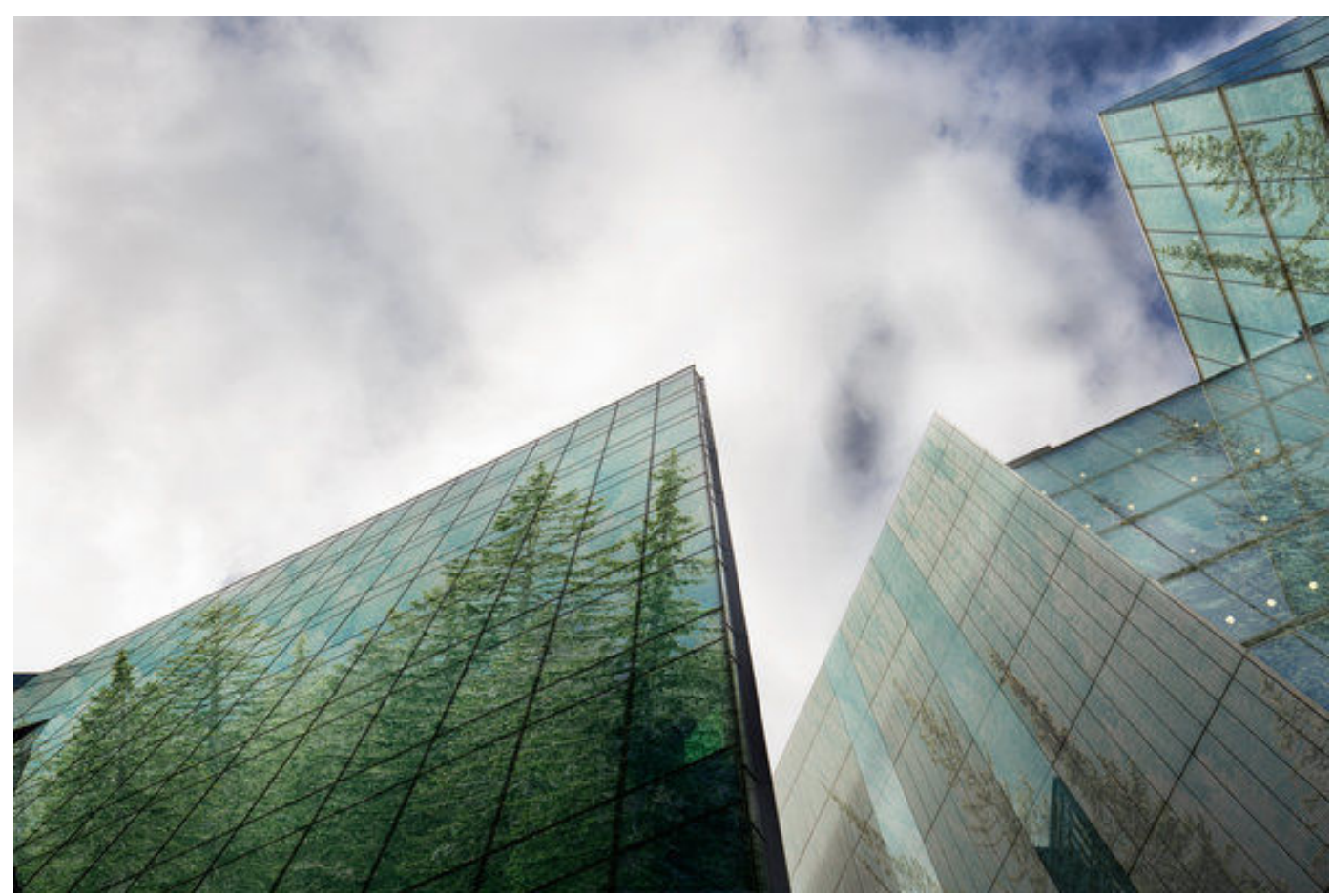

Sustainable investment: A new landscape

In today's changing global landscape, investors are increasingly seeking exposure to sustainable strategies. Examples of these include ESG funds and green bonds.

Since 2016, sustainable assets have increased by $34 \%$ globally. This remarkable growth can be explained by a number of complex drivers, including the wider availability of sustainability data and the higher number of responsible investing initiatives.

At the beginning of 2018, global sustainable assets reached US \$30.7 trillion. By region, the largest proportion of these assets was managed in Europe (46\%), followed by the United States (39\%), Japan and Canada (6-7\%, each).

Currently, more than 11,700 public companies disclose sustainability data, as measured by environmental, social and governance (ESG) metrics. Additionally, 
over 125 organisations, such as FTSE, MSCI and Sustainalytics, produce ESG research and ratings. Overall, broader access to ESG data has made it possible to integrate sustainability screens into the investment process.

A growing number of responsible investing initiatives, spurred by the Paris Climate Agreement and the UN's 2030 Agenda for Sustainable Development, as well as growing public concerns about climate change, are also helping to fuel asset flows into ESG-oriented strategies. In addition, policymakers around the world are introducing regulatory initiatives to help promote and define sustainable investing.

At this time, the most extensive proposal has come from the European Union. The European Commission has outlined an Action Plan on Sustainable Finance. This initiative aims to establish a clear classification system for sustainable activities, clarify asset managers' and institutional investors' responsibilities and integrate climate concerns into banks' risk management processes.

In the United States, the regulatory approach towards sustainable investing has been less prescriptive. In 2015, the US Labor Department (DOL) issued ESG Guidance, recognising that ESG issues can pose material risks to businesses. As such, the Department permits including ESG factors in investment policy statements, but disclosure is not mandatory.

Responsible finance initiatives have also gained traction in Asia. In China, listed companies will be required to disclose their ESG risks by 2020. Similarly, the Hong Kong Stock Exchange has also proposed to make ESG disclosures mandatory for public companies.

As sustainability data continues to proliferate and different international regulatory approaches keep being introduced, ESG vocabulary will likely become even more difficult to understand. To help clarify the confusion, we categorise sustainable investing strategies into four categories, namely ESG integration, portfolio screening, active ownership and impact investing.

\section{A lasting trend?}

Broadly speaking, investors who apply ESG integration aim to improve the risk and return profiles of their portfolios. For example, they may underweight or remove companies that have significant ESG-oriented risks. These include litigation, reputational and regulatory risks, which may lead to financial losses. Proponents of this approach may not be driven by a clear values mandate. Instead, they examine ESG-related data to enhance their existing investment goals.

Meanwhile, the portfolio screening approach selects companies based on specific criteria. This method adopts either an exclusionary or an inclusionary screening. An exclusionary screening underweights or removes companies if they don't 
meet investors' ESG requirements. For example, a portfolio that excludes oil and gas companies, such as Royal Dutch Shell and Exxon Mobil, adopts this approach.

Our chart shows that as the ESG exposure of sustainable equity exchange traded funds (ETFs) increases, the tracking error of the funds relative to the MSCI ACWI, a global stock index, rises in tandem.

Navigating the sustainable investing landscape can be complex and challenging yet also rewarding. As is almost always the case, investors need to determine their objectives, evaluate their options, decide on action and reassess their decision periodically. With public pressure mounting for action to address climate change, the global landscape for sustainable investment strategies should become even more promising.

COOECD Observer November 2019

\section{References}

Global Sustainable Investment Alliance (2018), 2018 Global Sustainable Investment Review http://www.gsi-alliance.org/wp-content/uploads/2019/03/ GSIR_Review2018.3.28.pdf

Grim, D. M. and D.B. Berkowitz (2018), ESG, SRI, and impact investing: A primer for decision-making, https://personal.vanguard.com/pdf/ISGESG.pdf

European Commission (2019), Green finance https://ec.europa.eu/info/businesseconomy-euro/banking-and-finance/sustainable-finance_en

US Department of Labor (2018), ESG Investment Considerations, in Field Assistance Bulletin No. 2018-01, http://dx.doi.org/https://www.dol.gov/ agencies/ebsa/employers-and-advisers/guidance/field-assistance-bulletins/ 2018-01

HKEX (2019), Review of the environmental, social and governance reporting guide and related listing rules https://www.hkex.com.hk/-/media/HKEXMarket/News/Market-Consultations/2016-Present/May-2019-Review-of-ESGGuide/Consultation-Paper/cp201905.pdf?la=en 